\title{
Disostosis cleidocraneal
}

\author{
Dres.: Humberto Broitman D.*, Sergia Mancinelli P,** y Ximena Gallegos A.*»*
}

\section{ABSTRACT}

\section{“CLEIDOCRANEAL DYSOSTOSIS"}

\begin{abstract}
The appearance of a clinic case of "Cleidocraneal dystostos" in the new bom unit of Pedintrics Departmenl in the "Guillermo Grant Benavente Hospital" (Concepción. Chile), made some physicians do a revision of this pathology. The case is described with the help of other studies by different guthors and is compared. It is then possible to test in this case the presence of the most typical sygmology of Cleidocraneal D. Even if the new born has samples of other genopaties it is not testable, because, as his parents, his cariogram is absolutely oormal (by "Bandeo" technique). All the clinis and radiologic sygnology of the case is described.
\end{abstract}

La primera descripción de este caso, hecha probablemente en 1776, por Morand (Francia). quien describió el examen de un niño nacido sin clavículas. ]

Fueron Marie y Santon en I897, quienes propusieron el nombre actualmente en uso, ${ }^{3}$ sin embargo es necesario considerar que esta Disostosis puede también afectar a otras porciones óseas (púbis, manos y a las piezas dentarias). El polimorfismo hace que a veces, incluso, esté ausente el defecto craneal o el clavicular. 3,4 . 5. 6

El primer caso publicado en Chile fue en 1944. por los doctores Hasbún y Meneghello.

Es una enfermedad que se transmite en forma autosómica dominante y con alta penetrancia ${ }^{3.4}$ 4, afecta por igual a ambos sexos. $3 .+$ Esta noxa empezaría a actuar desde el feto de 5 semanas, afectando tanto el desarrollo de los huesos membranosos como los cartilaginosos.

El paciente típico se caracteriza por presentar una gran cabeza, cara pequeña, cuello largo, hombros caidos, tórax angosto y talla corta. El cráneo posee una funtanela anterior muy amplia y cuyo cierre, al igual que el de las suturas (longitudinal y coronaria), que son anchas y deprimidas, ge realiza alrededor de los 16 años de edad. ${ }^{5}$ 7. 8. 9 El diámetro fronto occipital está acortado y el trangverso aumentado, la región frontal es protuberante; presentan prognatismo mandibular y un ensanchamiento de la base de la nariz. El paladar es alto y estrecho, ojival y a veces fisurado.

El cierre tardío de las suturas y fontanelas se realiza a veces con interposición de huesos Wormia+

* Residente Becario, Servicio Pediatría, Hospital Gmo. Grant Benavente.

**Prolesor de Genética, Ingtituto Ciencias Biológicas, Depto, de Fisiopatología, Universidad de Concepción.

${ }^{* * *}$ Médico General de Zona, Hospital de Coronel S.N.S. nos, dejando un cráneo deforme y con una depresión central. La agenesia de las clavículas es bilateral en el $82 \%$ de los casos. ${ }^{5 .} 7$ A veces la clavícula ausente, en forma parcial o total, está reemplazada por un cordón fibroso que a la radiología simula una fractura. ${ }^{3}$ La cirugía paliativa es necesaria cuando fragmentos claviculares comprimen algún nervio.

La agenesia pareial o total de las clavículas más la laxitud musculotendinosa, permite a los hombros acercarse a la línea media.

Muchas veces el hallazgo radiológico casual de la agenesia clavicular despierta la sospecha diagnóstica.

Con respecto a la dentición: la primera es normal, pero la definitiva es tardía y pueden haber variaciones en la forma y en el tamaño 3. 4. 5

La aplasia del púbis se presenta en el $10 \%$ de los casos, pero la mineralización retardada y el retraso en el cierre de la sinfisis es más frecuente. ${ }^{3}$ 4. 9 Los huesos de las manos y pies son pequeños.7 Se presentan con menos frecuencia otras malformaciones, por ejemplo xifosis.

Los exámenes de laboratorio son normales, y la radiología es la encargada de ratificar el diagnóstico. Los signos radiológicos van a depender de la extensión de las lesiones y de las edades de los pacientes en estudio.

Estos individuos en la edad adulta son generalmente de baja estatura y de inteligencia normal; pueden llevar una vida absolutamente normal sin resultar una carga para sus familiares.

Caso Clínico: J. C. C., N. ${ }^{\circ}$ de observación 771.479. Fecha de nacimiento 29 de mayo de 1978 . Hospital de Coronel. Cesárea a las 38 semanas de gestación por rotura prematura de membrana. Apgar 8 al nacer, 8 a los 3 y 5 minutos.

Antecedentes de embarazo: primigesta, no de- 
seado. Madre se colocó una ampolla de Tocofinal (m.r.).

Antecedentes familiares: ambos padres son sanos. Examen físico y radiológico negativo para disostosis. Tiene un tio materno con probable agenesia clavicular derecha (no se pudo examinar).

Al examen físico de ingreso se aprecia (Fots N.* 1): Peso $3100 \mathrm{~g}$. Talla $50 \mathrm{~cm}$. Cráneo 35,5 centímetros.

Fontonela anterior muy amplia, que abarca prácticamente toda la cabeza, en su porción superior. Las suturas muy anchas se encuentran deprimidas. Frente prominente. Globos oculares con tendencia al exoftalmo. Hipertelorismo. Puente nasal bajo.

\section{FOTO N.: 1}

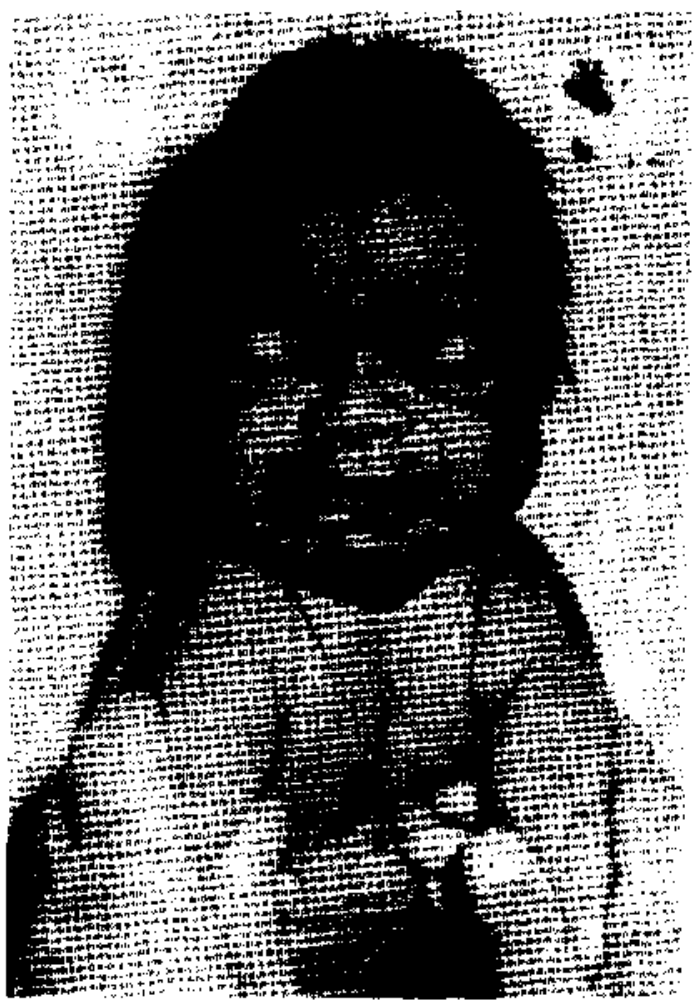

Cuello, no se palpan clavículas, a derecha un corto cordón fibroso.

En manos se aprecia pliegue palmar único bilateral y clinodactilia del primer dedo e implantación alta del quinto dedo.

Ambos pies presentan desviación interna de sus tercios anteriores, bilateral (pie adducto).

Siendo una enfermedad autosómica dominante con alta penetrancia, era dable encontrar antecedentes familiares positivos, los cuales no fueron encontrados en los padres, ni por radiología. pero exis- ten fuertes sospechas en un tío matemo que tiene un "hombro caido", desde su nacimiento, el cual no pudo ser examinado por residir fuera de la Zona.

Los exámenes de laboratorio, salvo los radiolóficos que en este caso, al igual que en todos los descritos por otros autores, confirman el diagnóstico. están en línites normales lo cual está descrito en la literatura. ${ }^{3,4}, 5$

El cariograma practicado a los padres y al niño fue normal. Este paciente es portador de pliegue simio bilateral, clinodactilia del primer dedo e implantación alta de ambos quintos dedos, lo que confirma que este examen puede resultar normal, habiendo anormalidad ósea.

Ninguna de las lesiones óseas de este niño son de solución quirúrgica: su pie adducto será tratado por ortopedista alrededor de los 10 años de edad, si tuviese problemas con los esbazos de clavículas. éstos podrían ser extírpados.

A la madre, a pesar de ser primípara y con remordimientos por el intento de aborto, se le ha instruido repetidamente para criar a su hijo, como un niño normal, debiendo guardar cuidado en el manejo de su cabeza (evitar golpes). Por ser estos niños de inteligencia normal es responsabilidad médica evitar la neurosis materna que desembocaría, a la larga, en un niño sobreprotegido e incapaz de solucionar los problemas que le deparará su medio ambiente. más adelante.

El hallazgo de un cráneo blando, en un recién nacido, plantea el diagnóstico diferencial de la disostosis cleidocraneal con osteogenesis imperfecta. condrodistrofia calcific ante y la hipofosfatasia 4 , pero en este caso la agenesia clavicular y la particular radiologia. tanto para el cráneo, claviculas o como para la desmineralización de la pelvis, más las otras patologías óseas (hemivértebras, pie adducto. falta de isquiones. etc.J, ayudaron a simplificar el diagnóstico.

\section{RESUMEN}

La presencia de un caso clínico de disostosis cleidocraneal, en el Servicio de Pediatría, Unidad de Recién Nacido del Hospital Guillermo Grant Benavente de Concepción. motiva la revisión de esta patología: se describe la clínica de esta enfermedad a la luz de lo escrito por diversos autores y se compara con nuestro caso, pudiéndose comprobar la presencia de la signología mâs tipica en nuestro caso. A pesar de tener manifestaciones de otras genopatías. esto no se logra comprobar, ya que tiene, al igual que sus 
padres cariograma toun técnica de bandeos) absolutamente nurmal: se describe tonda la signolsugia rlínica y radiolígica de este niñu estudiado.

\section{REFERENIIIAS}

IJackson. W, P., Osteu-Dental dyaplasia. Acta Med. Scand 134: 292. $196 \mathrm{I}$

${ }^{2}$ Hasbiu, J., Merneghello. J. Disosursig (.leiducraneal Rev. Chil Pediatr. 15: 675, 1944.

3Smith, D. W. Recugnizable patterns of humbn malformations W. B. Saunders cumpany, 1976.
\$Schaffer, A. J. Enfermedades del recién nacido, Salvat Editores, 1974 .

${ }^{5}$ Nelson, W. E. Tratade de pediatria, Salvar Editures. Tumo II. $1383,1931$.

6Forland M., Cleidereraneal Dysostosis, Am. J. Med. 33: 742. $1 \% 2$.

${ }^{7}$ Hexsinger, R. M., Orihopedic problems of the shoulder and nerk. Pediatric Clin. North An. 24: 889. 1977.

${ }^{b}$ Grossling, G. et al. Dysostosia Cleidueraneal. Areh. Soc. Circ. Cil. 13: 478, 196l.

"Caffey, 1. Peatiatrics X Rays Diagnosis. Year book Medical Publishers. 1967. 